(pp 306-311)

\title{
Influence of Subliminal Rhythm Shift with a Lengthened Interval on Reactive Periodic Movement
}

Yuki TAKAHASHI, PT, MS, Satoshi FUJIWARA, PT, MS, Masanori ITO, PT, PhD, Naoki KADO, PT, PhD Department of Physical Therapy, Kobe College of Rehabilitation and Welfare

Toshiaki SUZUKI, PT, DMSc

Graduate School of Kansai University of Health Sciences

Purpose: This study aimed to investigate the influence of interonset interval (IOI) shift below the threshold of conscious recognition on the control of reactive movements.

Methods: Thirteen healthy individuals (10 males, 3 females; mean age, $27.4 \pm 3.9$ years) were included in this study. All subjects performed reaction time tasks, wherein they were instructed to raise their right ankle in response to each auditory stimulus. There were three test conditions as follows: periodic auditory stimuli at an IOI of 1,500 ms and a shift in the last stimulus interval to $1,575 \mathrm{~ms}$ and $1,800 \mathrm{~ms}$, respectively, during successive stimuli sequences at an IOI of 1,500 ms. Electromyographic reaction time (EMG-RT) for the last stimulus was compared among the three conditions.

Result: The EMG-RT was significantly delayed under condition 3 compared with that under conditions 1 and 2. A comparison of conditions 1 and 2 revealed no significant differences.

Conclusion: Periodic rhythmic stimulation is predicted to comprise some time width, and reactive movements can be performed without delay under conditions with an IOI shift below the threshold of conscious recognition. 